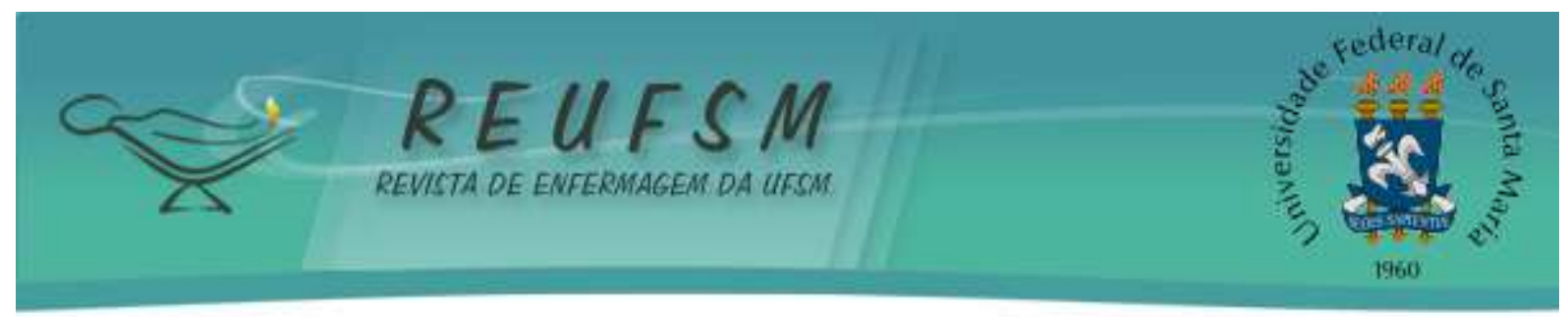

\title{
ARTIGO ORIGINAL FATORES ASSOCIADOS À ADESÃO AO TRATAMENTO ANTIRRETROVIRAL EM ADULTOS INFECTADOS PELO HIV: ESTUDO TRANSVERSAL
}

\author{
FACTORS ASSOCIATED TO THE ADHERENCE TO ANTIRETROVIRAL \\ TREATMENT IN HIV-INFECTED ADULTS: CROSS-SECTIONAL STUDY
}

\section{FACTORES ASOCIADOS CON A LA ADHESIÓN AL TRATAMIENTO ANTIRRETROVIRAL EN ADULTOS INFECTADOS POR EL VIH: ESTUDIO TRANSVERSAL}

\author{
Samuel Spiegelberg Zuge ${ }^{1}$ \\ Marcelo Ribeiro Primeira ${ }^{2}$ \\ Eduardo Remor ${ }^{3}$ \\ Tânia Solange Bosi de Souza Magnago ${ }^{4}$ \\ Cristiane Cardoso de Paula ${ }^{5}$ \\ Stela Maris de Mello Padoin ${ }^{6}$
} Doi: $10.5902 / 2179769225657$

RESUMO: Objetivo: analisar os fatores associados à adesão ao tratamento antirretroviral em adultos com HIV. Método: estudo transversal, com 179 adultos atendidos em serviço especializado, no Sul do Brasil. Foi utilizado o "Cuestionário para la Evaluación de la Adhesión al Tratamiento Antiretroviral" (CEAT-VIH) e análise estatística descritiva e inferencial (Regressão de Poisson). Resultados: dos participantes, 83,2\% foram classificados em não adesão ao tratamento. Estiveram associados à não adesão o menor nível de escolaridade, a dificuldade de manutenção do acompanhamento ambulatorial, a propensão para alcoolismo, a mudança no estilo de vida e a utilização de medicações psiquiátricas. Conclusões: o diagnóstico precoce de adesão possibilita o planejamento de ações educativas focadas nas características associadas à não adesão.

Descritores: HIV; Terapia antirretroviral de alta atividade; Adesão à medicação; Adulto; Enfermagem.

ABSTRACT: Aim: to analyze factors associated to the adherence to antiretroviral treatment in adults with HIV. Method: a cross-sectional study with 179 adults attending a specialized service in the South of Brazil. To assess adherence, the "Questionnaire for the Evaluation of Adhesion to Antiretroviral Treatment" (CEAT-VIH) was used. The results were evaluated with descriptive and inferential statistics (Poisson regression). Results: Among the

\footnotetext{
${ }^{1}$ Enfermeiro, Mestre em Enfermagem, Discente do Curso de Doutorado do Programa de Pós-Graduação em Enfermagem, Universidade Federal de Santa Maria, Docente da Universidade do Oeste de Santa Catarina, São Miguel do Oeste (SC), Brasil. E-mail: samuelzuge@gmail.com

2 Enfermeiro, Discente do Curso de Mestrado do Programa de Pós-Graduação em Enfermagem, Universidade Federal de Santa Maria, Santa Maria, Rio Grande do Sul, Brasil. E-mail: mrp_sm@ hotmail.com

${ }^{3}$ Psicólogo, Doutor em Psicologia, Professor Adjunto no Instituto de Psicologia e Pós-Graduação em Psicologia da Universidade Federal do Rio Grande do Sul, Porto Alegre, RS. E-mail: eduardo.remor@ufrgs.br

4 Enfermeira, Doutora em Enfermagem, Docente no Programa de Pós-Graduação em Enfermagem da Universidade Federal de Santa Maria, Santa Maria, RS, Brasil. E-mail: tmagnago@terra.com.br

5 Enfermeira, Doutora em Enfermagem, Docente no Programa de Pós-Graduação em Enfermagem da Universidade Federal de Santa Maria. Santa Maria, RS, Brasil. E-mail: cris_depaula1@ @otmail.com

6 Enfermeira, Doutora em Enfermagem, Docente no Programa de Pós-Graduação em Enfermagem da Universidade Federal de Santa Maria. Santa Maria, RS, Brasil. E-mail: stelamaris_padoin@ hotmail.com
} 


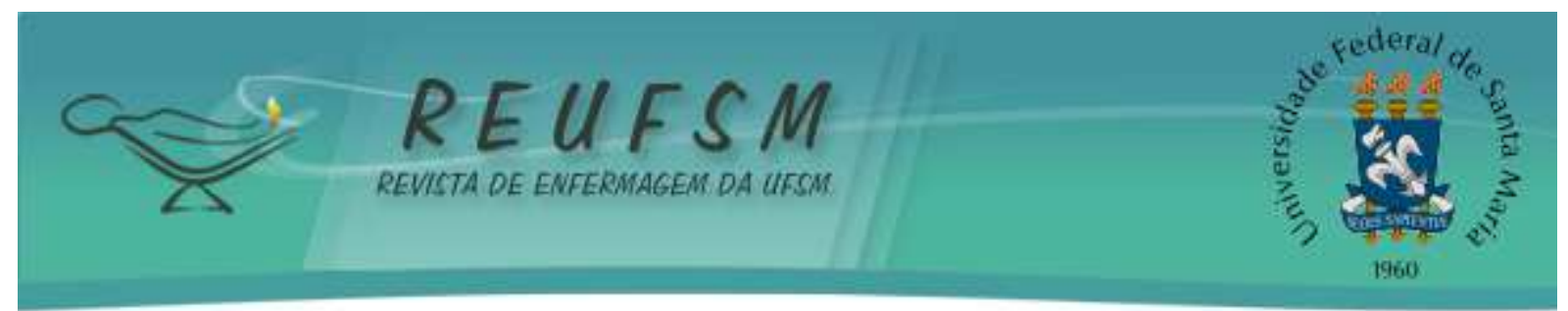

participants, $83.2 \%$ were defined as non-adherent to antiretroviral therapy. Lower level of schooling, difficulty in maintaining health service attendance, propensity for alcoholism, change in lifestyle and use of psychiatric medications were associated with noncompliance. Conclusions: the early diagnosis of adherence allows the planning of educational actions focused on the characteristics associated with non-adherence.

Descriptors: HIV; Antiretroviral therapy, highly active; Medication adherence; Adult; Nursing.

RESUMEN: Objetivo: examinar los factores asociados a la adhesión al tratamiento antirretroviral en adultos con VIH. Método: estudio transversal, con 179 adultos, que asisten a un servicio de salud especializado en el sur de Brasil. Se utilizó el "Cuestionario para la Evaluación de la Adhesión al Tratamiento Antirretroviral" (CEAT-VIH) para evaluar la adhesión. Los resultados fueron analizados por medio de estadística descriptiva e inferencial (Regresión de Poisson). Resultados: entre los participantes 83,2\% fueron clasificados como no adhesión al tratamiento. Se asoció a la no adhesión el bajo nivel de escolaridad, la dificultad de manutención de la atención en el ambulatorio, la propensión para el alcoholismo, el cambio del estilo de vida y la utilización de medicamentos psiquiátricos. Conclusiones: el diagnóstico precoz de la adhesión posibilita la planificación de acciones educativas específicas para las características de la no adhesión.

Descriptores: VIH; Terapia antirretroviral altamente activa; Adhesión al tratamiento; Adulto; Enfermería.

\section{INTRODUÇÃO}

A epidemia da infecção pelo Vírus da Imunodeficiência Humana (HIV) apresentou diversas demandas assistenciais para a saúde. Entre estas, a distribuição de medicamentos gratuitos por meio do Sistema Único de Saúde (SUS) do Brasil. Mas existem desafios que perpassam o tratamento da infecção, como a adesão. Definida como o grau em que o usuário segue a prescrição médica, está implicada com o comportamento terapêutico, que interfere no uso de medicamentos, o qual deve ser negociado, planejado e avaliado. ${ }^{1-3}$

A avaliação da adesão é objeto de estudo de muitas pesquisas ${ }^{4-10}$ e seus resultados têm evidenciado que os usuários possuem dificuldades de adesão, as quais repercutem em sua vida. Isso implica na condição clínica, qualidade de vida e no âmbito de suas relações sociais. Também repercutem negativamente na capacidade dos sistemas de saúde de alcançarem as metas de saúde da população. ${ }^{4-9}$

Entre as metas, com o objetivo de minimizar os efeitos da epidemia do HIV no mundo proposto pela Joint United Nations Program on HIV/AIDS (UNAIDS), espera-se que até 2030 90\% das pessoas infectadas pelo HIV saibam de seu diagnóstico, que $90 \%$ das pessoas diagnosticadas estejam em tratamento e que $90 \%$ das pessoas em tratamento apresentem 


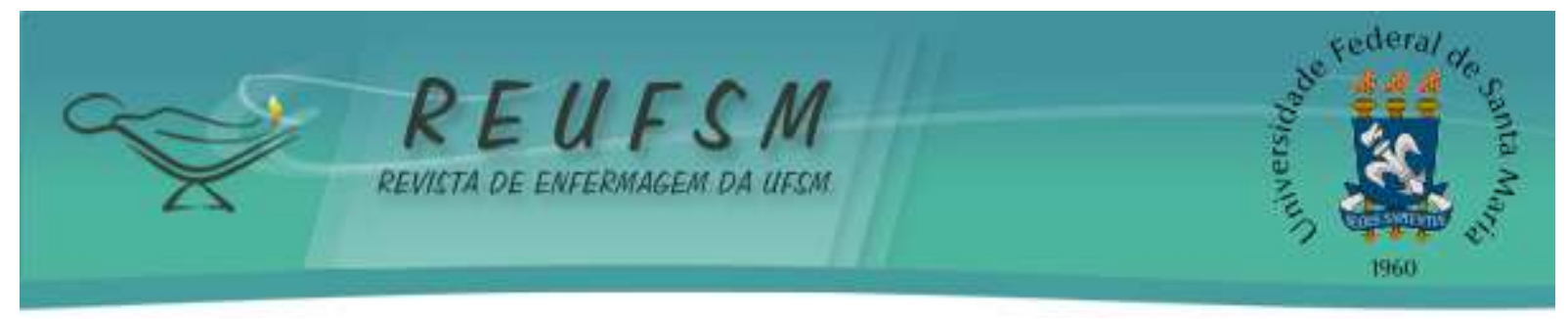

supressão viral. A supressão viral é uma ferramenta importante na prevenção do HIV e está diretamente relacionada com a adesão ao tratamento. ${ }^{10}$

A avaliação da adesão pode ser desenvolvida de forma contínua e permanentemente nos serviços de saúde. Atentando para abranger o controle das questões clínicas e os fatores comportamentais das pessoas em tratamento. Assim, indica-se a avaliação da adesão para além da ingestão medicamentosa, considerando a influência das características sociodemográficas, clínicas e comportamentais. ${ }^{4-9}$ Entende-se que, ampliar estudos acerca da prevalência e as implicações da não adesão ainda são necessários, uma vez que poderá ser focalizada a busca de medidas que venham a melhorar as condições de saúde e qualidade de vida desta população.

Para promover as ações educativas, a enfermagem deve estar atenta a este contexto com vistas à diminuição de potenciais riscos para o sucesso do tratamento. Ao considerar sua inserção em uma equipe de saúde multiprofissional, destaca-se sua especificidade ao desenvolver o acolhimento dos usuários e o importante papel nas ações educativas para o autocuidado na adesão. $^{11}$

Este estudo tem como pergunta de pesquisa: As características sociodemográficas, clínicas e comportamentais estão associadas à adesão ao tratamento antirretroviral? E como objetivo analisar os fatores associados à adesão ao tratamento antirretroviral em adultos com HIV.

\section{MÉTODO}

Trata-se de um estudo transversal, desenvolvido em um Ambulatório de Doenças Infecciosas que é referência para municípios da região centro-este do Rio Grande do Sul, Brasil. A seleção dos participantes foi a amostragem por conveniência, conforme a marcação de consulta no ambulatório, no período de janeiro a julho de 2012. Para o cálculo do tamanho da amostra assumiu-se uma proporção estimada de 50\%, erro amostral de 5,7\% e 95\% de confiança, de uma população de 432 pessoas, sendo a amostra composta por 179 participantes.

Como critérios de inclusão: realizar acompanhamento neste serviço; retirar os medicamentos antirretrovirais (ARVs) na Unidade de Dispensação de Medicamentos deste serviço; estar em tratamento há pelo menos três meses (nos primeiros meses os efeitos colaterais dos medicamentos podem ser fatores de confundimento para a avaliação da 


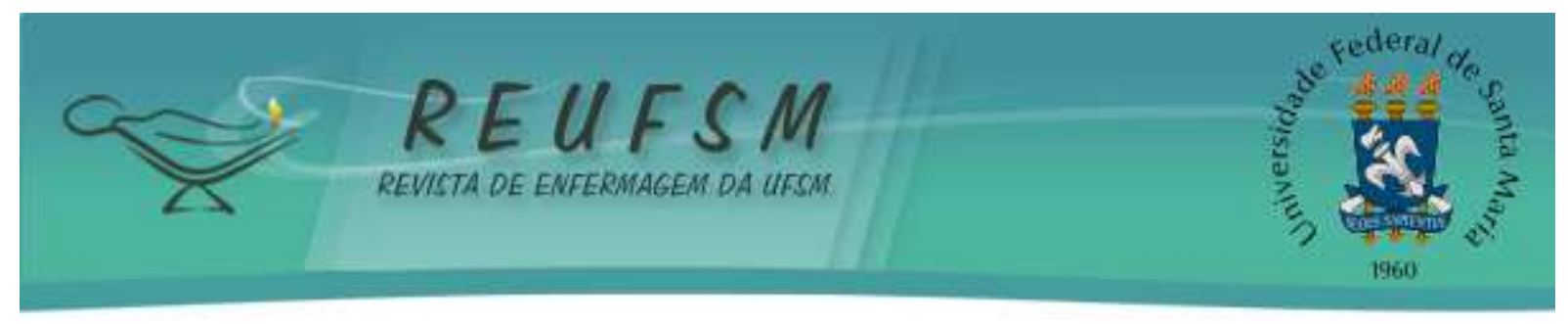

adesão); e ter 20 anos ou mais. Foram excluídos indivíduos pertencentes ao sistema prisional (a fim de não interferir no princípio de privacidade); e mulheres em período gravídico puerperal (uma vez que poderiam estar iniciando o tratamento, como medida de profilaxia para a transmissão vertical do HIV).

Para avaliar a adesão ao tratamento antirretroviral (desfecho) foi utilizado o "Cuestionário para la Evaluación de la Adhesión al Tratamiento Antiretroviral" (CEATVIH). Este questionário avalia a perspectiva multidimensional da adesão, considera o comportamento de ingestão dos medicamentos antirretrovirais, e também as dificuldades e barreiras percebidas pela pessoa para cumprir o tratamento. É constituído de 20 itens, que em conjunto refletem uma avaliação global da adesão ao tratamento. ${ }^{5,7}$

Para analisar as demais características (variáveis independentes), foi elaborado um instrumento em três seções: a) sociodemográficas: sexo, idade, nível de escolaridade, número de filhos, situação marital, situação empregatícia, renda per capita familiar; b) clínicas: doença oportunista, tempo de diagnóstico, tempo de tratamento; c) comportamentais: compartilhamento do seu diagnóstico no ambiente de trabalho, utilização de álcool e/ou outras drogas (lícitas/ilícitas), manutenção do acompanhamento no serviço de saúde, participação em grupo de apoio, avaliação da propensão para o alcoolismo (CAGE), ${ }^{12}$ mudança no estilo de vida, interferência na ingesta da medicação por mudança na prescrição, interferência na ingesta da medicação por estar em jejum, utilização de medicações psiquiátricas.

Os dados foram inseridos no programa Epi-info®, versão 3.5, por meio de dupla digitação independente. Após correção de erros e inconsistências, foi realizada análise no programa PASW Statistics ${ }^{\circledR}$ (Predictive Analytics Software, da SPSS Inc., Chicago - USA) versão 18.0 for Windows.

Todas as variáveis foram, inicialmente, analisadas por estatística descritiva (média e desvio padrão; frequência absoluta e relativa), dependendo do tipo de variável. A avaliação da adesão foi obtida pela soma de todos os itens do CEAT-VIH (valor mínimo possível 17, valor máximo possível 89). Quanto maior a pontuação, maior o grau de adesão. ${ }^{7}$ Neste estudo, o grau de adesão foi definido como adesão (escore bruto $\geq 83$; percentil $\geq 85$ ) e não adesão (escore bruto < 83; percentil < 85). A consistência interna do CEAT-VIH foi avaliada por meio do coeficiente Alpha de Cronbach $(\alpha=0,78)$ e o teste de normalidade apontou uma distribuição assimétrica dos escores no instrumento, segundo o teste Kolmogorov-Smirnov. 


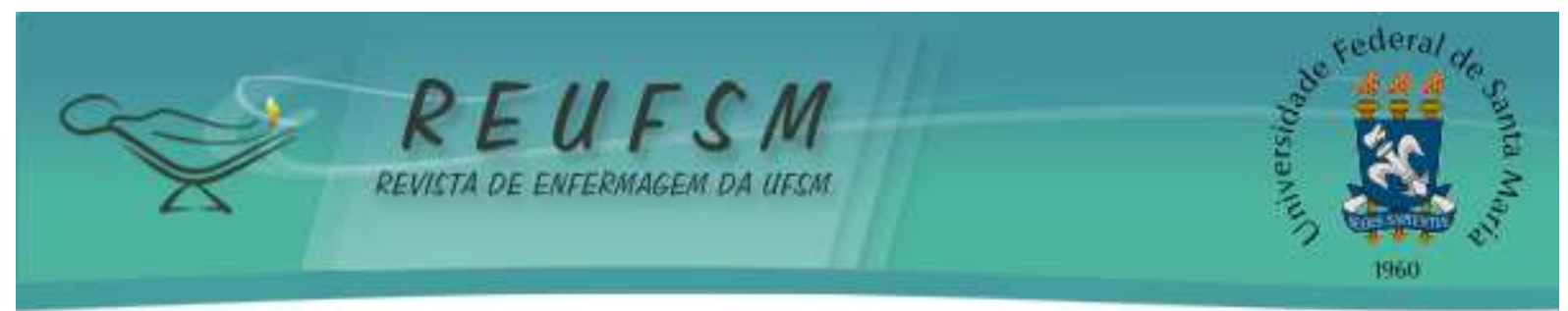

Após, para a análise dos fatores associados à adesão, utilizou-se o Teste do QuiQuadrado de Pearson ou o Exato de Fisher. As variáveis que apresentaram $p<0,10$ foram incluídas no modelo multivariado, analisado por regressão de Poisson. Nesta análise, a medida de efeito foi a Razão de Prevalência (RP) e seus respectivos intervalos de confiança (IC95\%). Em todas as análises, foi considerada diferença significativa entre os grupos avaliados quando $\mathrm{p}<0,05$.

Obteve-se aprovação do Comitê de Ética em Pesquisa da instituição pelo CAEE: 0322.0.243.000-11. A participação da população do estudo foi voluntária, mediante Consentimento Livre Esclarecido, e a pesquisa foi conduzida de acordo com os padrões éticos exigidos.

\section{RESULTADOS}

Dos 179 adultos participantes da pesquisa, 91 (50,8\%) eram do sexo masculino, 131 $(73,2 \%)$ referiram cor ou raça branca, $65(36,3 \%)$ com idade entre 40 e 49 anos, idade média 43,2, ( $\pm 10,48), 101(56,4 \%)$ não haviam estudado ou tinham estudado até o ensino fundamental, $76(42,4 \%)$ tinham de dois a quatro filhos, $92(51,4 \%)$ relataram que sua situação conjugal era que viviam sozinhos, $117(65,4 \%)$ não estavam empregados no momento, 127 (73,0\%) com renda per capita familiar de até dois salários mínimos e para 122 $(68,2 \%)$ a forma de transmissão do HIV foi a sexual (Tabela 1).

Segundo a avaliação do CEAT-VIH, 149 (83,2\%) foram classificados em não adesão ao tratamento antirretroviral. Na relação com os dados sociodemográficos, a adesão apresentou uma associação estatisticamente significativa quando relacionada ao nível de escolaridade (Tabela 1).

Tabela 1 - Distribuição dos participantes, segundo variáveis sociodemográficas e adesão ao tratamento antirretroviral. Santa Maria/RS, Brasil, 2012

\begin{tabular}{lcccc}
\hline & Variáveis & Adesão & Não Adesão & p \\
\cline { 3 - 4 } & & $\mathbf{N}(\%)$ & $\mathbf{N}(\%)$ & \\
\hline Sexo & & & & \\
$\quad \begin{array}{l}\text { Masculino } \\
\text { Feminino }\end{array}$ & $91(50,8)$ & $16(17,6)$ & $75(82,4)$ & 0,764 \\
Idade & $88(49,1)$ & $14(15,9)$ & $74(84,1)$ & \\
$\quad$ De 20 a 43 anos & $92(51,4)$ & $13(14,1)$ & $79(85,9)$ & 0,333
\end{tabular}




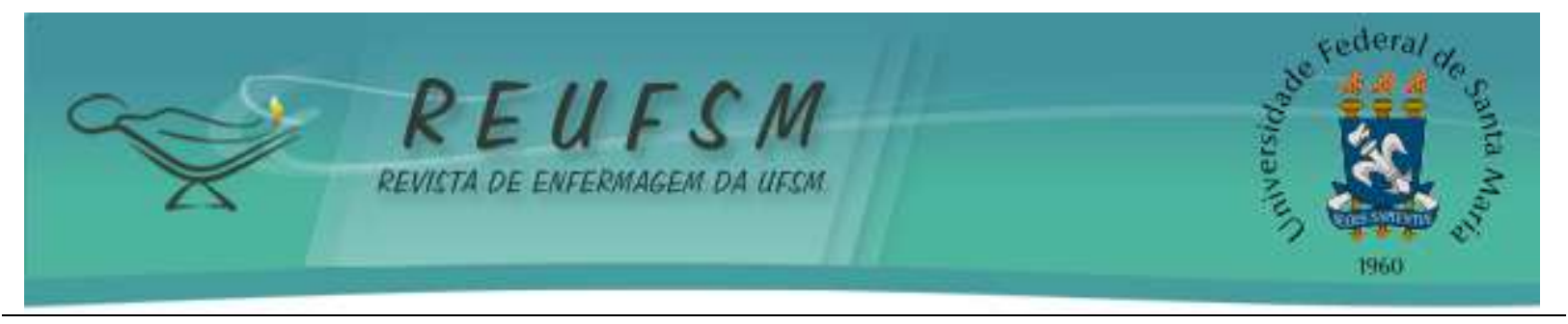

\begin{tabular}{|c|c|c|c|c|}
\hline \multirow{2}{*}{ Variáveis } & \multirow{2}{*}{$\mathbf{N}(\%)$} & Adesão & Não Adesão & \multirow{2}{*}{$\mathbf{p} \dagger$} \\
\hline & & $\mathbf{N}(\%)$ & $\mathbf{N}(\%)$ & \\
\hline Maior e igual a 44 anos & $87(48,6)$ & $17(19,5)$ & $70(80,5)$ & \\
\hline $\begin{array}{l}\text { Nível de Escolaridade } \\
\text { Não estudou ou estudou até } \\
\text { ensino fundamental } \\
\text { Até o ensino médio } \\
\text { Até o ensino superior }\end{array}$ & $\begin{array}{l}101(56,4) \\
45(25,1) \\
33(21,2)\end{array}$ & $\begin{array}{l}12(11,9) \\
6(13,3) \\
12(36,4)\end{array}$ & $\begin{array}{l}89(88,1) \\
39(86,7) \\
21(63,6)\end{array}$ & $\mathbf{0 , 0 0 4}$ \\
\hline $\begin{array}{l}\text { Número de filhos } \\
\text { Nenhum filho } \\
\text { Um filho } \\
\text { Dois a quatro filhos } \\
\text { Cinco ou mais filhos }\end{array}$ & $\begin{array}{l}38(29,1) \\
52(42,4) \\
76(7,3) \\
13(7,3)\end{array}$ & $\begin{array}{c}5(13,2) \\
14(26,9) \\
10(23,2) \\
1(7,7)\end{array}$ & $\begin{array}{l}33(86,8) \\
38(73,1) \\
66(86,8) \\
12(92,3)\end{array}$ & 0,128 \\
\hline $\begin{array}{l}\text { Situação Marital } \\
\text { Vive com esposo(a)/ } \\
\text { companheiro(a) } \\
\text { Vive sozinho(a) }\end{array}$ & $\begin{array}{l}87(48,6) \\
92(51,4)\end{array}$ & $\begin{array}{l}16(18,4) \\
14(15,2)\end{array}$ & $\begin{array}{l}71(81,6) \\
78(84,8)\end{array}$ & 0,147 \\
\hline $\begin{array}{l}\text { Situação empregatícia } \\
\text { Empregado } \\
\text { Não Empregado }\end{array}$ & $\begin{array}{c}62(34,6) \\
117(65,4)\end{array}$ & $\begin{array}{l}10(16,1) \\
20(17,1)\end{array}$ & $\begin{array}{l}52(83,9) \\
97(82,9)\end{array}$ & 0,869 \\
\hline $\begin{array}{l}\text { Renda per capita familiar * } \\
\text { Até dois salários mínimos } \\
\text { Mais de dois salários } \\
\text { mínimos }\end{array}$ & $\begin{array}{c}127(73) \\
47(27)\end{array}$ & $\begin{array}{l}17(13,4) \\
12(25,5)\end{array}$ & $\begin{array}{c}110(86,6) \\
35(74,5)\end{array}$ & 0,056 \\
\hline
\end{tabular}

* N=174; Valor do salário mínimo - R\$ 622,00 e US\$ 1156,92.

$\dagger$ Teste Qui-Quadrado de Pearson.

A relação entre as características clínicas e a adesão ao tratamento não apontou relação estatística significativa (Tabela 2).

Tabela 2 - Distribuição dos participantes, segundo variáveis clínicas e adesão ao tratamento antirretroviral. Santa Maria/RS, Brasil, 2012

\begin{tabular}{|c|c|c|c|c|}
\hline \multirow{2}{*}{ Variáveis } & \multirow{2}{*}{$\mathbf{N}(\%)$} & Adesão & Não Adesão & \multirow{2}{*}{$\mathbf{p} \dagger$} \\
\hline & & $\mathbf{N}(\%)$ & $\mathbf{N}(\%)$ & \\
\hline \multicolumn{5}{|l|}{ Doença oportunista } \\
\hline Apresentou & $79(44,1)$ & $11(13,9)$ & $68(86,1)$ & \multirow{2}{*}{0,367} \\
\hline Não apresentou & $100(55,9)$ & $19(19,0)$ & $81(81,0)$ & \\
\hline \multicolumn{5}{|l|}{ Tempo de diagnóstico } \\
\hline Menor ou igual a 5 anos & $60(33,5)$ & $9(15,0)$ & $59(85,0)$ & \multirow{3}{*}{0,553} \\
\hline De 6 a 10 anos & $68(38,0)$ & $14(20,6)$ & $54(79,4)$ & \\
\hline Mais de 11 anos & $51(28,5)$ & $7(13,7)$ & $44(86,3)$ & \\
\hline \multicolumn{5}{|l|}{ Tempo de tratamento* } \\
\hline Menor ou igual a 5 anos & $85(48,0)$ & $13(15,3)$ & $72(84,7)$ & \multirow{3}{*}{0,162} \\
\hline De 6 a 10 anos & $65(36,7)$ & $15(23,1)$ & $50(76,9)$ & \\
\hline Mais de 11 anos & $27(15,3)$ & $2(7,4)$ & $25(92,6)$ & \\
\hline
\end{tabular}

* $\mathrm{N}=177$.

$\dagger$ Teste Qui-Quadrado de Pearson. 


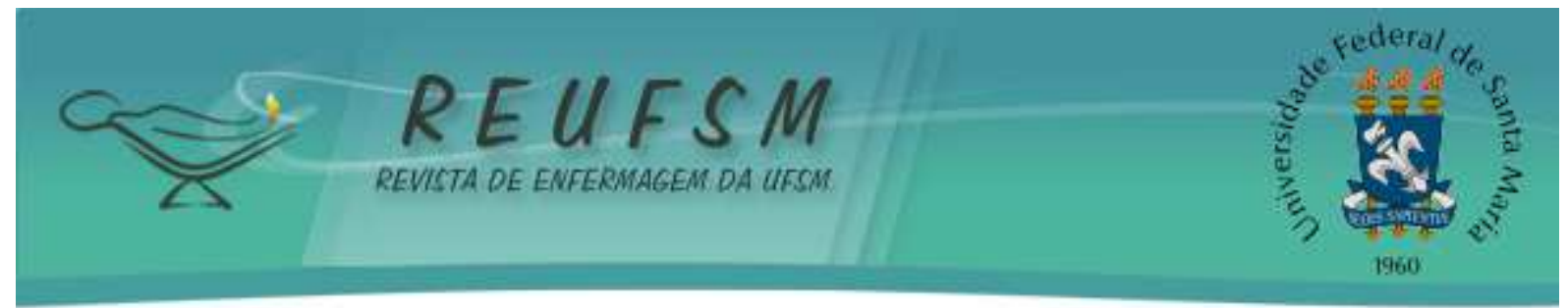

Na relação com as características comportamentais, a adesão apresentou significância estatística quando relacionada com a "manutenção do acompanhamento no serviço de saúde", a "avaliação da propensão para alcoolismo (CAGE)", a "mudança no estilo de vida" e a "utilização de medicações psiquiátricas" (Tabela 3).

Tabela 3 - Distribuição dos participantes, segundo variáveis comportamentais e adesão ao tratamento antirretroviral. Santa Maria/RS, Brasil, 2012

\begin{tabular}{|c|c|c|c|c|}
\hline Variáveis & $\mathbf{N}(\%)$ & $\begin{array}{c}\text { Adesão } \\
\mathbf{N}(\%)\end{array}$ & $\begin{array}{l}\text { Não Adesão } \\
\mathbf{N}(\%)\end{array}$ & $\mathbf{p}$ \\
\hline \multicolumn{5}{|l|}{ Uso álcool/drogas (lícitas/ilícitas) } \\
\hline Sim & $14(7,8)$ & $1(7,1)$ & $13(92,9)$ & \multirow[t]{2}{*}{$0,470 \dagger$} \\
\hline Não & $165(92,2)$ & $29(17,6)$ & $136(82,4)$ & \\
\hline \multicolumn{5}{|c|}{$\begin{array}{l}\text { Manutenção do acompanhamento no serviço de } \\
\text { saúde: }\end{array}$} \\
\hline Apresenta dificuldades & $75(41,9)$ & $6(8,0)$ & $69(92,0)$ & \multirow[t]{2}{*}{$\mathbf{0 , 0 0 8 *}$} \\
\hline Apresenta facilidades & $104(58,1)$ & $24(23,1)$ & $80(76,9)$ & \\
\hline \multicolumn{5}{|l|}{ Participação em grupo de apoio } \\
\hline Sim & $13(7,3)$ & $1(7,7)$ & $12(92,3)$ & \multirow[t]{2}{*}{$0,476 \dagger$} \\
\hline Não & $166(92,7)$ & $29(17,5)$ & $137(82,5)$ & \\
\hline \multicolumn{5}{|l|}{ Propensão para o alcoolismo $(n=48)$} \\
\hline Não etilista & $30(62,5)$ & $7(23,3)$ & $23(76,7)$ & \multirow[t]{2}{*}{$\mathbf{0 , 0 3 6 \dagger}$} \\
\hline Propensão para o alcoolismo & $18(37,5)$ & $0(-)$ & $18(100,0)$ & \\
\hline \multicolumn{5}{|l|}{ Mudança no estilo de vida } \\
\hline $\mathrm{Sim}^{5}$ & $78(43,6)$ & $8(10,3)$ & $70(89,7)$ & \multirow[t]{2}{*}{$0,041 *$} \\
\hline Não & $101(56,4)$ & $22(21,8)$ & $79(78,2)$ & \\
\hline \multicolumn{5}{|l|}{ Uso de medicações psiquiátricas } \\
\hline Sim & $29(16,2)$ & $9(30,0)$ & $20(70,0)$ & \multirow[t]{2}{*}{$\mathbf{0 , 0 3 2} *$} \\
\hline Não & $150(83,8)$ & $21(14,0)$ & $129(86,0)$ & \\
\hline
\end{tabular}

* Teste Qui-Quadrado de Pearson.

$\dagger$ Teste Exato de Fisher.

$\mathrm{Na}$ análise de associação entre a adesão e demais variáveis, foi possível identificar que aqueles que não estudaram ou estudaram até o ensino fundamental (RPaj2: 1,53; IC95\%: 1,10-2,12) e os que estudaram até o ensino médio (RPaj2: 1,49; IC95\%: 1,05-2,12) tiveram prevalências mais elevadas de não adesão (Tabela 4).

Tabela 4 - Análises bruta e ajustada entre adesão ao tratamento antirretroviral, variáveis sociodemográficas, comportamentais e clínicas. Santa Maria/RS, Brasil, 2012

\begin{tabular}{|c|c|c|c|c|c|c|c|c|c|}
\hline \multirow[b]{2}{*}{ Variáveis } & \multicolumn{9}{|c|}{ Adesão ao tratamento } \\
\hline & $\mathbf{R P b} *$ & IC $95 \%$ & $\mathbf{p}$ & RPaj1 $\dagger$ & $\begin{array}{c}\text { IC } \\
95 \%\end{array}$ & $\mathbf{p}$ & $\begin{array}{c}\text { RPaj2 } \\
+\end{array}$ & IC95\% & $\mathbf{p}$ \\
\hline \multicolumn{10}{|c|}{ Nível de Escolaridade } \\
\hline $\begin{array}{l}\text { Não } \\
\text { estudou } \\
\text { ou } \\
\text { ensino }\end{array}$ & 1,15 & $1,03-1,28$ & 0,010 & 1,11 & $\begin{array}{c}0,99- \\
1,24\end{array}$ & 0,054 & 1,53 & $1,10-2,12$ & $\begin{array}{c}\mathbf{0 , 0 1} \\
\mathbf{0}\end{array}$ \\
\hline
\end{tabular}




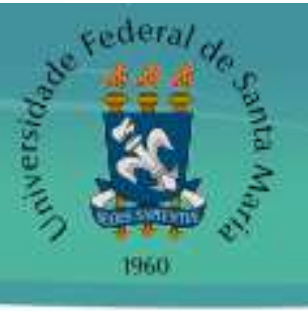

\begin{tabular}{|c|c|c|c|c|c|c|c|c|c|}
\hline \multicolumn{10}{|l|}{ fundamental } \\
\hline $\begin{array}{l}\text { Ensino } \\
\text { médio }\end{array}$ & 1,14 & $1,02-1,23$ & 0,023 & 1,12 & $\begin{array}{c}0,99- \\
1,25\end{array}$ & 0,054 & 1,49 & $1,05-2,12$ & $\begin{array}{c}0,02 \\
6\end{array}$ \\
\hline $\begin{array}{l}\text { Ensino } \\
\text { superior }\end{array}$ & 1 & - & - & 1 & - & - & 1 & - & - \\
\hline Manutenção do & comp & mento no se & o de saú & & & & & & \\
\hline Dificuldades & 1,08 & $1,03-1,15$ & 0,004 & & & & 1,32 & $0,98-1,77$ & $\begin{array}{c}0,06 \\
4\end{array}$ \\
\hline Facilidades & 1 & - & - & & & & 1 & - & - \\
\hline Avaliação da p & pensã & a o alcoolis & & & & & & & \\
\hline $\begin{array}{l}\text { Propensão } \\
\text { ao } \\
\text { alcoolismo }\end{array}$ & 1,13 & $1,04-1,23$ & 0,005 & & & & 0,96 & $0,84-1,10$ & $\begin{array}{c}0,57 \\
3\end{array}$ \\
\hline Não etilista & 1 & - & - & & & & 1 & - & - \\
\hline Mudança no es & lo de v & & & & & & & & \\
\hline Sim & 1,06 & $1,00-1,13$ & 0,032 & & & & 0,79 & $0,59-1,05$ & $\begin{array}{c}0,10 \\
9\end{array}$ \\
\hline Não & 1 & - & - & & & & 1 & - & - \\
\hline Utilização de $n$ & dicaçõ & siquiátricas & & & & & & & \\
\hline Sim & 1,10 & $0,99-1,22$ & 0,070 & & & & 1,34 & $0,81-2,21$ & $\begin{array}{c}0,25 \\
3\end{array}$ \\
\hline Não & 1 & - & - & & & & 1 & - & - \\
\hline
\end{tabular}

* RPb: Razão de Prevalência bruta.

$\dagger$ RPaj1: Razão de Prevalência ajustada para Adesão, escolaridade e renda per capita familiar.

$\ddagger$ RPaj2: Razão de Prevalência ajustada para adesão, escolaridade, compartilhamento do seu diagnóstico no ambiente de trabalho, manutenção do acompanhamento no serviço de saúde, utilização de medicações psiquiátricas, mudança no estilo de vida, e avaliação da propensão para o alcoolismo.

\section{DISCUSSÃO}

Os resultados deste estudo apresentaram prevalência para não adesão (83,2\%), o que indica a preocupação com esta população e a necessidade de avaliação contínua incluindo fatores relacionados, considerando a possibilidade de variações entre as populações em diferentes períodos. Estudos de revisão sistemática e meta-análise evidenciam que a adesão ao tratamento antirretroviral é altamente variável entre as populações, com taxas de cumprimento entre 20 e $100 \% .^{2,5}$

Outras pesquisas que utilizaram o CEAT-VIH identificaram índices de população em não adesão que variaram entre $78,3 \%$ no Estado da Paraíba, ${ }^{4} 70,3 \%$ na região Agreste de Pernambuco, ${ }^{13} 69,3 \%$ no Estado de São Paulo ${ }^{3}$ e 51,3\% no Estado do Ceará. ${ }^{14}$ Em outros países tem-se: Guiné Equatorial ${ }^{15} \operatorname{com} 55 \%$, Peru ${ }^{16} \operatorname{com} 74,5 \%$, na Colômbia ${ }^{8}$ e Romênia ${ }^{6}$ são 


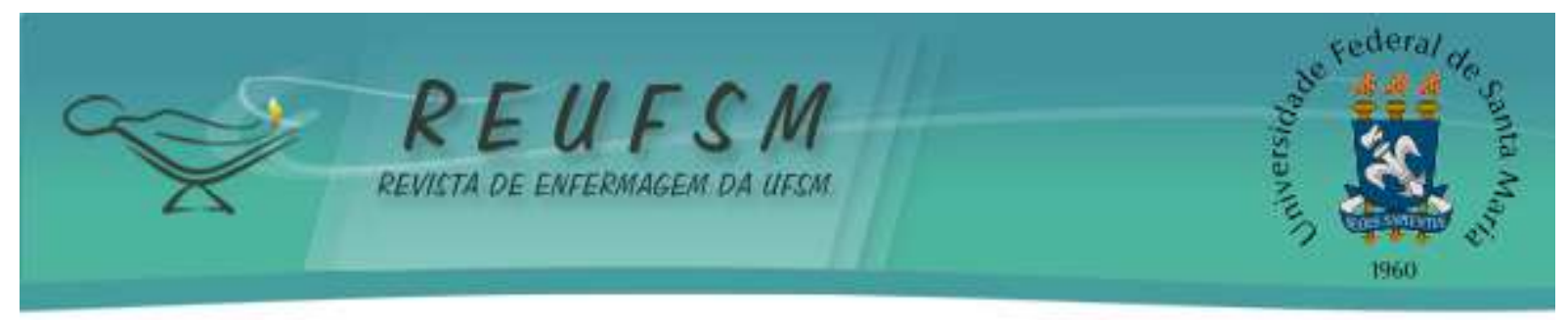

igualmente $15 \%$ de adesão estrita. Tais resultados mantêm a condição da adesão como um problema de saúde pública de âmbito mundial.

Dentre os fatores que apresentaram relação com a não adesão foi evidenciada a escolaridade. Esta, em determinadas situações, pode estar associada à renda per capita, o que implica no acesso a bens e consumo, como o acesso e o seguimento do tratamento de saúde, bem como a falta de compreensão acerca da gravidade da condição crônica de saúde, especialmente em fase assintomática. As pessoas com nível baixo de escolaridade podem estar predispostas a maior dificuldade de entendimento do tratamento e suas instabilidades, ou ainda apresentar dificuldades de acessar recursos ou informações acerca do tratamento. Desta forma, constata-se que maior tempo de estudo está associado a uma boa adesão.,13,17,18

Outro resultado que esteve associado à não adesão foi o fato de considerarem difícil manter o acompanhamento de sua saúde no serviço ambulatorial. As dificuldades podem ser inúmeras, como as relacionadas aos meios de transporte municipais e intermunicipais, os aspectos social e pessoal como a modificação de sua rotina de trabalho. O que implica nas condições de lidar com a condição crônica de saúde, com demandas de assistência à saúde e alterações no cotidiano por longo tempo. ${ }^{18}$

Neste estudo, as alterações no cotidiano vão além do acompanhamento ambulatorial, implicam em necessidade de mudança do estilo de vida. Esta foi uma variável que obteve resultado significativo quando relacionada com a adesão. Diante desta evidência, o tópico deverá ser abordado nas intervenções da enfermagem, pautadas nas implicações que o usuário poderá ter a partir do diagnóstico, mais especificamente quando iniciar a terapia, incluindo os aspectos que se referem ao regime terapêutico complexo e aos novos hábitos de cuidado da saúde. ${ }^{5,19}$

Foi considerada como um preditor na avaliação da adesão a propensão para o alcoolismo, o que permite inferir que estes adultos tendem a não realizar corretamente o tratamento devido ao uso de álcool. O uso nocivo do álcool é um problema de saúde pública que traz consequências negativas não só para o indivíduo, mas para sua a família e a sociedade. O baixo consumo de bebidas alcoólicas não traz repercussões negativas relacionadas à adesão, no entanto, constata-se que o abuso desta substância pode alterar a qualidade de vida, fator determinante para a continuidade do tratamento. Fica claro que, ao ser identificado o comportamento de consumo de risco para essa substância, as orientações de 


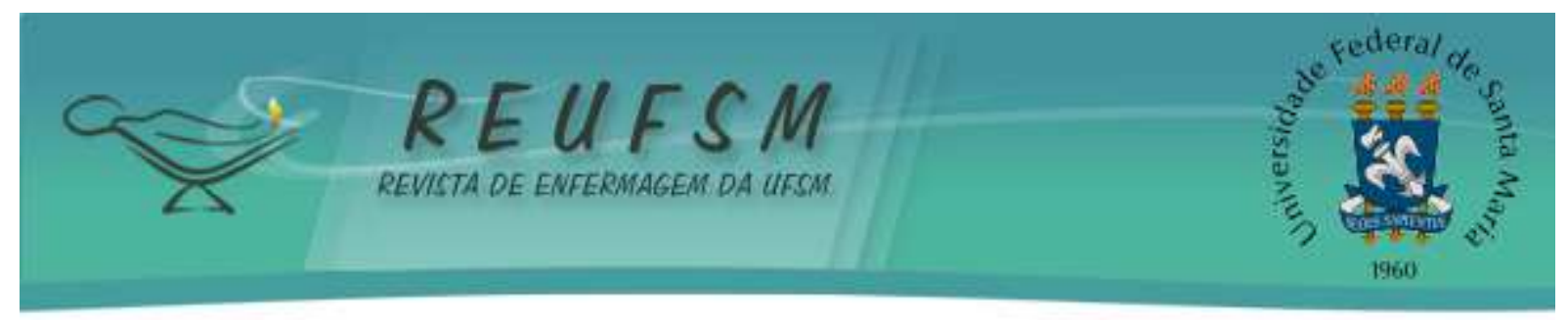

enfermagem deverão incluir os riscos do uso nocivo e a possibilidade da redução de danos, além do apoio social a esta população. ${ }^{20}$

A questão do uso de medicamentos psiquiátricos obteve resultado significativo quando relacionada com a adesão. Esta situação implica no direcionamento das ações educativas que a enfermagem poderá programar para este usuário, uma vez que irá se somar a demanda específica da infecção pelo HIV com a situação de algum transtorno psiquiátrico. Foram identificadas a depressão e a ansiedade como preditores da não adesão., ${ }^{5,19}$

A única variável que manteve associação, com diferença estatística entre os grupos de adesão e não adesão, foi o nível de escolaridade. As demais variáveis perderam a associação na análise ajustada por fatores de confusão (Regressão de Poisson). No entanto, essas variáveis podem ser consideradas importantes na avaliação realizada pelos profissionais da saúde, devido à possibilidade de identificação de características que evidenciem uma possibilidade de não adesão.

Destaca-se que, dentre as características sociodemográficas, clínicas e comportamentais avaliadas neste estudo, não foi possível identificar convergências e divergências com os estudos encontrados na literatura que utilizaram o CEAT-VIH, uma vez que os estudos não realizaram estas associações, sendo este o diferencial deste estudo. No entanto, aponta-se que a variável escolaridade tem sido avaliada sistematicamente em estudos que utilizaram o CEAT-VIH, ${ }^{3,5,13}$, nos quais pessoas com um menor nível de escolaridade apresentavam escores associados à não adesão. Com esta evidência, a equipe multiprofissional poderá estabelecer estratégias de detecção precoce de indivíduos com risco de não adesão, ou possíveis candidatos a programas de intervenção para melhorar os níveis de adesão e autocuidado em saúde.

\section{CONCLUSÕES}

Foram identificadas evidências das características sociodemográficas, clínicas e comportamentais associadas à adesão de modo negativo quando: há baixo nível de escolaridade, dificuldade de manutenção do acompanhamento ambulatorial, o uso de bebidas alcoólicas, dificuldades de mudança no estilo de vida e uso de medicamentos psiquiátricos, o que ratifica a adesão como um fenômeno multidimensional.

Na prática clínica indica-se a necessidade de somar esforços entre os diferentes atores para que se utilize sistematicamente este instrumento (CEAT-VIH), uma vez que se mostrou útil na 


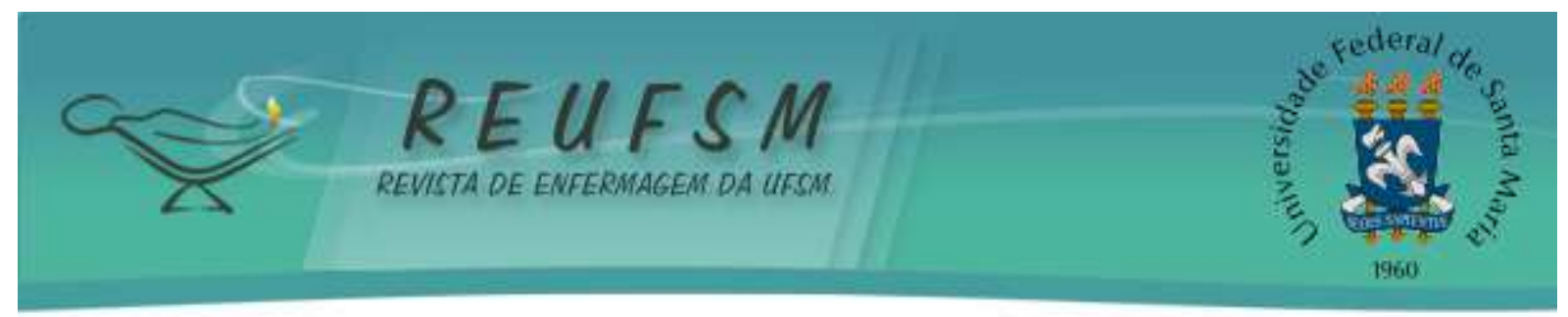

classificação dos níveis de adesão na população estudada. Poderá auxiliar os profissionais em formação (no ensino) ou em qualificação (na assistência) na identificação precoce da não adesão, fornecendo subsídios para um plano de ação e medidas promotoras de maior adesão.

Especificamente para área da enfermagem, tais ações poderão ser no âmbito individual com consultas de adesão ou no âmbito coletivo na forma de grupo operativo com os usuários. Estes com objetivo e aplicabilidade focada no processo de adesão e suas implicações, por exemplo, aquelas aqui evidenciadas. Sendo imprescindível o desenvolvimento do protagonismo deste usuário, no sentido de optar por sua participação ativa no tratamento e mudanças no estilo de vida.

O presente estudo não está isento de limitações, que precisam ser consideradas na generalização dos resultados para populações diferentes das descritas neste trabalho. Importante também considerar que a amostra foi coletada de forma não probabilística, formada de indivíduos pertencentes a uma região específica do país e que tinham acesso aos serviços públicos de saúde, o que indica a ampliação de pesquisas que contemplem as diversidades regionais e da assistência nos serviços de saúde.

\section{REFERÊNCIAS}

1. Fiuza MLT, Lopes EM, Alexandre HO, Dantas PB, Galvão MTG, Pinheiro AKB. Adesão ao tratamento antirretroviral: assistência integral baseada no modelo de atenção às condições crônicas. Esc Anna Nery Rev Enferm [Internet]. 2013 [acesso em 2017 set 18];17(4):740-8. Disponível em: http://dx.doi.org/10.5935/1414-8145.20130019.

2. Kahana SY, Rohan J, Allison S, Frazier TW, Drotar D. A meta-analysis of adherence to antiretroviral therapy and virologic responses in HIV-infected children, adolescents, and young adults. AIDS Behav [Internet]. 2013 [acesso em 2017 set 18];17(1):41-60. Disponível em: https://doi.org/10.1007/s10461-012-0159-4.

3. Dagli-Hernandez C, Lucchetta RC, Nadai TR, Galduróz JCF, Mastroianni PC. Selfperception of knowledge and adherence reflecting the effectiveness of antiretroviral therapy. Patient Prefer Adherence [Internet]. 2016 [acesso em 2017 set 18];10:1787-93. Disponível em: https://doi.org/10.2147/PPA.S112108.

4. Silva ACO, Reis RK, Nogueira JA, Gir E. Quality of life, clinical characteristics and treatment adherence of people living with HIV/AIDS. Rev Latinoam Enferm [Internet]. 2014 [acesso em 2017 set 18];22(6):994-1001. Disponível em: http://dx.doi.org/10.1590/01041169.3534.2508.

5. Remor E. Systematic Review of the Psychometric Properties of the Questionnaire to Evaluate the Adherence to HIV Therapy (CEAT-VIH). Patient [Internet]. 2013 [acesso em 2017 set 18];6(2):61-73. Disponível em: https://doi.org/10.1007/s40271-013-0009-0. 


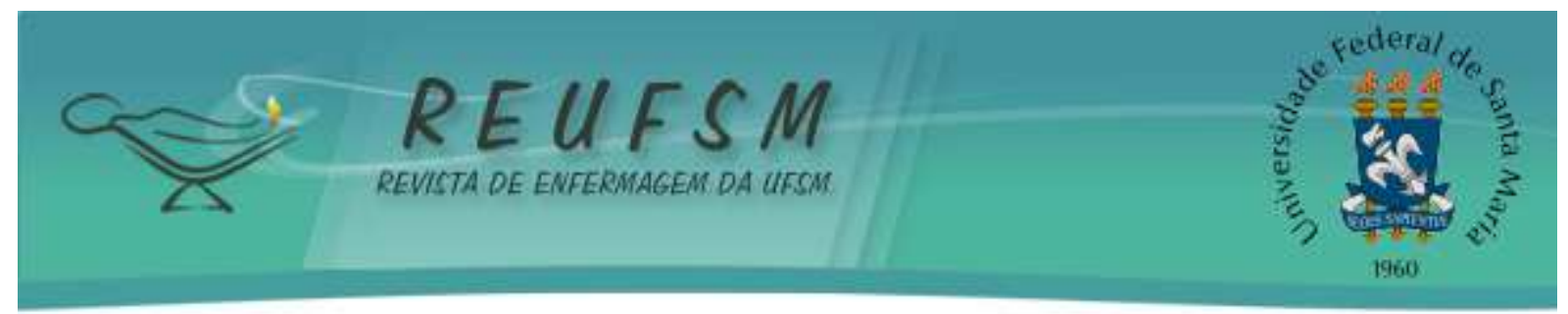

6. Dima AL, Schweitzer AM, Diaconita R, Remor E, Wanless RS. Adherence to ARV medication in Romanian young adults: self-reported behaviour and psychological barriers. Psychol Health Med [Internet]. 2013 [acesso em 2017 set 18];18(3):343-54. Disponível em: http://dx.doi.org/10.1080/13548506.2012.722648.

7. Remor E, Milner-Moskovics J, Preussler G. Adaptação brasileira do "Cuestionario para la Evaluación de la Adhesión al Tratamento Antiretroviral". Rev Saúde Pública [Internet]. 2007 [acesso em 2017 set 18];41(5):685-94. Disponível em: http://dx.doi.org/10.1590/S003489102006005000043.

8. Remor E. Self-reported adherence to antiretroviral therapy in HIV+Colombian population. sage open [Internet]. 2013 [acesso em 2017 set 18];3(3):1-7. Disponível em: https://doi.org/10.1177/2158244013497727.

9. Huang L, Li L, Zhang Y, Li H, Li X, Wang H. Self-efficacy, medication adherence, and quality of life among people living with HIV in hunan province of China: a questionnaire survey. J Assoc Nurses AIDS Care [Internet]. 2013 [acesso em 2017 set 18];24(2):145-53. Disponível em: http://dx.doi.org/10.1016/j.jana.2012.04.006.

10. UNAIDS. 90-90-90: uma meta ambiciosa de tratamento para contribuir para o fim da epidemia de AIDS. Geneva: UNAIDS; 2015.

11. Fernandes IA, Barbaglia J, Daniel KCS; Mello SSP. Orientação a pessoa vivendo com HIV: o papel do enfermeiro na adesão ao tratamento e no desenvolvimento da prática do autocuidado. Rev Fafibe [Internet]. 2015 [acesso em 2017 set 18];8(1):359-70. Disponível em: http://unifafibe.com.br/revistasonline/arquivos/revistafafibeonline/sumario/36/30102015190552 .pdf.

12. Williams N. The CAGE questionnaire. Occup Med [Internet]. 2014 [acesso em 2017 set 18];64:473-4. Disponível http://nationalpaincentre.mcmaster.ca/documents/cage_questionnaire.pdf.

13. Moraes DCA, Oliveira RC, Motta MCS, Ferreira OLC, Andrade MS. Terapia antirretroviral: a associação entre o conhecimento e a adesão. Rev Pesqui Cuid Fundam [Internet]. 2015 [acesso em 2017 set 18];7(4):3563-73. Disponível em: http://saudepublica.bvs.br/pesquisa/resource/pt/bde-27211.

14. Galvão MTG, Soares LL, Pedrosa SC, Fiuza MLT, Lemos LA. Quality of life and adherence to antiretroviral medication in people with HIV. Acta Paul Enferm [Internet]. 2015 [acesso em 2017 set 18];28(1):48-53. Disponível em: http://dx.doi.org/10.1590/19820194201500009.

15. Salmanton-García J, Herrador Z, Ruiz-Seco P, Nzang-Esono J, Bendomo V, Bashmakovic E, et al. Self-reported adherence to antiretroviral therapy in HIV+ population from Bata, Equatorial Guinea. AIDS Care [Internet]. 2015 [acesso em 2017 set 18];28(5):543-53. Disponível em: http://dx.doi.org/10.1080/09540121.2015.1124976.

16. Tello-Velásquez JR, Díaz-Llanes BE, Mezones-Holguín E, Rodrígues-Morales AJ, Huamaní C, Hernández AV, et al. La mala calidad de sueño se asocia a uma menor adherencia al tratamiento antirretroviral de gran actividad em pacientes peruanos com infección por VIH/SIDA. Cad Saúde Pública [Internet]. 2015 [acesso em 2017 set 18];31(5):989-1002. Disponível em: http://dx.doi.org/10.1590/0102-311X00010014. 


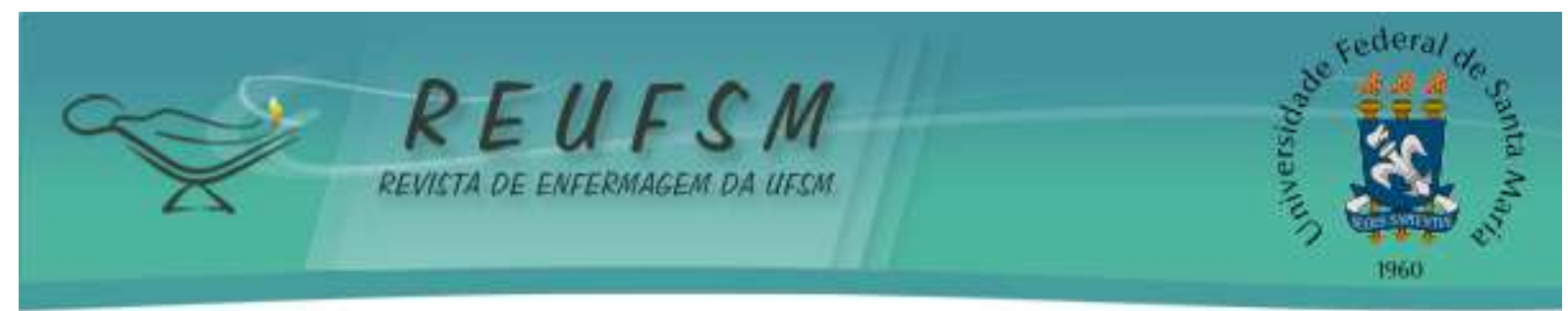

17. Foresto JS, Melo ES, Costa CRB, Antonini M, Gir E, Reis RK. Adesão à terapêutica antirretroviral de pessoas vivendo com HIV/aids em um município do interior paulista. Rev Gaúcha Enferm [Internet]. 2017 [acesso em 2017 set 11];38(1):e63158. Disponível em: http://dx.doi.org/10.1590/1983-1447.2017.01.63158.

18. Padoin SMM, Paula CC, Zuge SS, Primeira MR, Santos EEP, Tolentino LC. Fatores associados à não adesão ao tratamento antirretroviral em adultos acima de 50 anos que têm HIV/Aids. DST J Bras Doenças Sex Transm [Internet]. 2011 [acesso em 2017 set 18];23(4):194-7. Disponível em: http://www.dst.uff.br/revista23-42011/9.Fatores\%20associados\%20a\%20nao\%20adesao\%20ao\%20tratamento.pdf.

19. Boery RNSO, Santos NA, Boery EN, Cassotti CA, Maia VM, Silva JSL, et al. Fatores que interferem na adesão dos portadores de AIDS aos antirretrovirais, Jequié, Bahia, Brasil. Rev Saúde Com [Internet]. 2015 [acesso em 2017 set 18];11(3):233-42. Disponível em: http://www.uesb.br/revista/rsc/v11/v11n3a03.pdf.

20. Santos VF, Galvão MTG, Cunha GH, Lima ICV, Gir E. Alcohol effect on HIV-positive individuals: treatment and quality of life. Acta Paul Enferm [Internet]. 2017 [acesso em 2017 set 18];30(1):94-100. Disponível em: https://dx.doi.org/10.1590/1982-0194201700014.

\section{AGRADECIMENTOS}

Aos órgãos de fomento: Fundação de Amparo a Pesquisa do Rio Grande do Sul - FAPERGS e Conselho Nacional de Desenvolvimento Científico - CNPq pela Chamada Universal MCTI/CNPq (Processo: 478824/2012-2), pelos fomentos de IC e custeios.

Bolsistas de Iniciação Científica: pelo empenho e dedicação na coleta e digitação dos dados da pesquisa: Érika Eberlline Pacheco dos Santos, estudante do Curso de Graduação em Enfermagem da UFSM - Bolsista do PROBIC/FAPERGS; e Ilivelton Martins Koglin (in memoriam - vítima da tragédia em boate de Santa Maria, em 2013) estudante do Curso de Graduação em Enfermagem da Faculdade Integrada de Santa Maria - FISMA - Bolsista Voluntário.

Data de submissão: 20/01/2017

Data de aceite: 16/10/2017

Autor correspondente: Stela Maris de Mello Padoin

Endereço: Universidade Federal de Santa Maria - Avenida Roraima, n. 1000. Prédio 26, sala 1336. Bairro Camobi, Cidade Universitária, Santa Maria, RS, Brasil.

CEP: 97105-900.

Email: stelamaris_padoin@ @otmail.om 